\title{
LECTIN ACTIVITY IN PLANT ORGANS AT SYMBIOSIS FORMATION of ALFALFA WITH SINORHIZOBIUM MELILOTI UNDER DIFFERENT WATER CONDITIONS
}

\author{
Mykhalkiv L.M. \\ Institute of Plant Physiology and Genetics NAS of Ukraine, \\ 31/17, Vasylkivska Str., Kyiv, 03022 \\ E-mail: mykhalkiv@mail.ru
}

The paper covers study of insufficient water supply effect on lectin activity in different organs of alfalfa plants inoculated with Sinorhizobium meliloti 441 (active strain) and SHM1-48 (inactive strain) during the early stages of symbiosis formation and its active functioning. The correlations between the changes of lectin activity in alfalfa plants influenced by different water supply and strain characteristics, extent of plant development and stage of symbiosis formation.

Keywords: lectin activity, alfalfaa, Sinorhizobium meliloti, water supply.

Application of scientific research findings on biological nitrogen fixation into the modern agricultural practices can reduce the cost of energy and raw materials for the production of mineral nitrogen fertilizers and provide an ecologically clean production. At introduction of nitrogen-fixing plant-microbial systems into the agrocoenoses the impact of environmental factors on their formation, functioning and productivity should be taken into the account. Therefore, studies of resistance mechanisms of symbiotic systems to external stress as well as search for the adaptation means to harsh environmental conditions remain important. In this view, the role of plant lectins is little-studied, but is promising.

External factors of abiotic nature (drought, salinity, temperature fluctuations, heavy metals, etc.) can cause greater genes expression that are responsible for lectins synthesis, increasing the level of these proteins in plants and changing their properties [1-4]. Obviously, the accumulation of lectins is a general plant response to the different types of stress, however, the ways of their involvement to the adaptation processes remain unclear. O. Timofeeva et al. [5] have proved the involvement of cell wall lectins into the non-specific defense mechanisms of plants. It was shown that during the initial stage of formation of non-specific defense response in plants a rapid changes of lectins activity and composition in the cell wall take place, which depends on the functioning of calcium signaling system and may be associated with the role of lectins in signal transduction into the cell or in apoplast space. A direct relationship between the activity of cell wall lectin and the extend of winter wheat resistance to biotic and abiotic stressors was established, together with genetically determined and organ-specific correlation between the level of cell lectins activity and structural condition of cytoskeleton. Pattern for $\mathrm{Ca}^{2+}$ signaling pathway and cytoskeleton structures role in the regulating mechanism of lectins activity in the formation of adaptive responses of plants was suggested. Findings on possible cryoprotection effect of galactospesific lectins on isolated thylakoid membranes of chloroplasts are also of a big interest [6]. Due to the relative hydrophobicity, these lectins can bind to membrane glycolipids, strengthen thylakoid membrane structures and reduce their fluidity caused by damage during freezing. It was also shown [7] that induction of 
nonspecific resistance of legumes may be ensured by regulation of protective inhibition stage in general adaptation syndrome due to the activation of protein proteinase inhibitors and lectin-type proteins, which leads to a decrease of stressors toxic effect on nitrogen fixing system and plant productivity. Soluble lectins can function as elicitors [8], be involved in the regulation of gene expression in plants under stress by modulating O-GlcNAc-dependent transduction signals through specific protein-carbohydrate interactions with regulatory cytoplasmic and nuclear glycoproteins [9].

Taking into the account lectins role as an important factor of efficient symbiosis [10], as well as component of nonspecific plant response to stressors [11] the determination of lectins activity in alfalfa plants influenced by rhizobia inoculation of various activity under the optimal and insufficient water supply conditions was performed.

Materials and methods. The studies were conducted in greenhouse conditions at the Institute of Plant Physiology and Genetics, NAS of Ukraine. Alfalfa plants of Nadezhda cultivar were grown in sand culture (10 plants / pot) in a $4 \mathrm{~kg}$ Wagner containers (washed river sand, moisture content - 60\% TMC) at natural lighting, temperature and humidity. Mineral nutrition supply was prepared according to Hellriegel with 0.25 dose of nitrogen and addition of trace elements B, Mo and Fe. Prior to sowing seeds were sterilized with concentrated sulfuric acid, washed in running tap water and inoculated with two strains of Sinorhizobium meliloti nodule bacteria - 441 (active) and CXM1-48 (inactive). Bacterial cultures were grown on solid mannitol-yeast medium at a temperature of $28{ }^{\circ} \mathrm{C}$ for 4 days. Duration of seeds inoculation -1 hour, titer of inoculation suspension $-10^{9}$ cells $/ \mathrm{ml}$.

On the $7^{\text {th }}$ (experiment $\# 1$, trifoliate leaf emergence) or $20^{\text {th }}$ day (experiment $\# 2$, shooting stage) the appropriate plants were exposed to drought conditions $(30 \%$ TMC) by controlled irrigation. Analysis samples were taken: in experiment $\# 1$ - after 3 and 10 days after exposure to drought conditions, while in experiment $\# 2-$ on 3,7 and 11 day of drought.

Nitrogen fixation activity of root nodules was determined with acetylene reduction method [12] on gas chromatograph "Agilent 6850". Biological repetition 5 times. Vegetative plants mass ( $\mathrm{g}$ dry matter/ plant) was measured with $10-15$ biological repetition.

Phytohemagglutins (lectins) were isolated from roots, stems and leaves of alfalfa plants using ethanol elution method [13], with minor modification. The plant material was grinded in a porcelain mortar with the addition of $0.5 \mathrm{M}$ phosphate buffer ( $\mathrm{pH}$ 5.0), used as extraction solution. Extraction was carried out for 20 hours in a cool dark place. After that, mixture was centrifuged at $3000 \mathrm{rev} / \mathrm{min}$ for $10 \mathrm{~min}$. Supernatant was decanted and mixed with $96 \%$-s' ethyl alcohol so that the output alcohol content in the mixture was $76 \%$. Received solution was centrifuged at 6000 $\mathrm{rev} / \mathrm{min}$ for $15 \mathrm{~min}$. Supernatant was poured out while the precipitate of protein was dissolved in saline. Determination of lectin activity in the received protein solution was performed by hemagglutination reactions in immunological plates with U-shaped holes [14]. Lectin activity was determined after samples incubation with human red blood cells a solution during 2 hours at room temperature corresponding to the biggest dilution, where hemagglutination was observed. 
The results of experiments were statistically analyzed using standard method [15]. Figure presents the arithmetic means and their standard errors.

Results and discussion. On the third day of plants cultivation under different water conditions (10-day plant) neither nodules on the roots of alfalfa plants or molecular nitrogen fixation activity were observed, although, as is well known [16], that this period of plant growth is characterized with an active internal changes in the structure and functioning of root caused by bacterial infection. Study of lectin activity in alfalfa plants inoculated with active strain 441 have shown the inhibition of erythrocyte sedimentation in the variants with insufficient water supply, while no changes of lectin activity in the plants inoculated with inactive strains were observed. No differences were revealed in plants inoculated with different strains (441 and CXM1-48) under optimal water supply.

After 10 days of plants growing under different water conditions the nodules formation was observed on the roots of alfalfa plants in variants with $60 \% \mathrm{TMC}$. At this, inoculation with $S$. meliloti 441 in some plants resulted in formation of botryoidal nodules. It should be noted that $S$. meliloti strain CXM1-48 have formed about $2-2.5$ times more nodules on the plant roots than the active strain 441 .

Evaluation of nitrogen fixation activity revealed minor ethylene peaks in the variant inoculated with $S$. meliloti 441 on optimal water supply background. As a result, of drought the significant reduction of dry plants matter was observed during this period, while no significant differences were noticed between the plants inoculated with different strains, under the same water conditions (Figure).

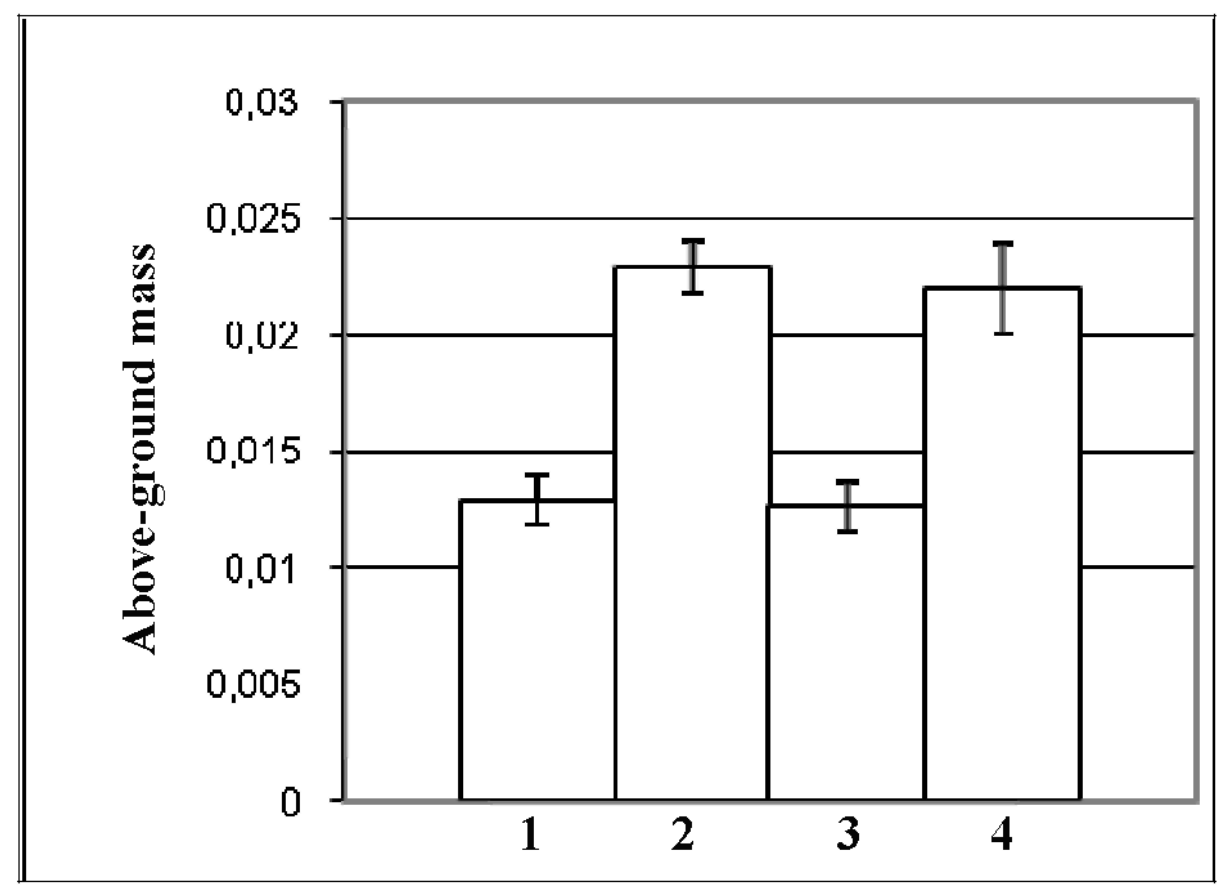

FIGURE. Above-ground mass (g dry matter / plant) of 17-day old alfalfa plants inoculated with different strains of Sinorhizobium meliloti on the background of optimal and low (10-day drought) water conditions (experiment \#1): 1 - inoculation with S. meliloti CXM1-48, 30\% TMC; 2 - S. meliloti inoculation with CXM1-48, $60 \%$ TMC; 3 - inoculation with S. meliloti 441, 30\% TMC; 4 - inoculation with S. meliloti 441, 60\% TMC. 
Estimation results of lectin activity in the roots and vegetative parts of the plants during this period are presented in Table 1. It was established that neither different levels of moisture nor nitrogen fixation activity of symbiosis have affected this index in plant roots. However, in vegetative mass the increase of lectin activity in variants with SHM1-48 strain application on both levels of water supply compared to the inoculation with 441 strain was observed.

TABLE 1. Lectin activity $(\mathrm{OA} / 50 \mathrm{ml})$ in 17-day old alfalfa plants at different levels of water supply (10-day drought) and inoculation with Sinorhizobium meliloti (experiment 1)

\begin{tabular}{|l|c|c|}
\hline \multicolumn{1}{|c|}{ Variants } & Root & Aboveground parts \\
\hline $\begin{array}{l}\text { S.meliloti CXM1-48, 30\% } \\
\text { TMC }\end{array}$ & 2 & 4 \\
\hline $\begin{array}{l}\text { S.meliloti CXM1-48, 60\% } \\
\text { TMC }\end{array}$ & 2 & 4 \\
\hline S.meliloti $441,30 \%$ TMC & 2 & 2 \\
\hline S.meliloti $441,60 \%$ TMC & 2 & 2 \\
\hline
\end{tabular}

Taking into the account that lectin activity is directly related to the nitrogen fixation activity [17] the opposite effect of inoculation with studied strains would be expected. Obviously, in this case, the influence of another factor, like, for example, the nodulation activity of the strain, which in S. meliloti SHM1-48 is higher than that of $S$. meliloti 441, or the culture characteristics or physiological peculiarities of the early stages of symbiosis formation have played the determining role.

Studying the impact of moisture deficit on the lectin activity in alfalfa plants at later stages of ontogenesis (early drought - shooting stage, 20-day old plants) the decrease of lectin activity in leaves of plants at $3^{\text {rd }}, 7^{\text {th }}$ and $11^{\text {th }}$ days of drought were observed on the background of inoculation with inactive strain SHM1-48 (Table 2). AS for the inoculation with 441 strain the lectin activity in leaves of plants with low water supply have increased after 3 days of drought, if compared to $60 \% \mathrm{TMC}$, while after 11 days, on the contrary - have decreased.

Regardless of inoculant strain, the increase of lectin activity in plant roots on water deficit background was observed.

In plant stems the influence of 3-day water deficit was noticed in variants with both strains - lectin activity was higher in 2 times. At $7^{\text {th }}$ day of drought it was established that in plants inoculated with inactive strain the lectin activity in stems was higher, regardless of the water conditions. But, after $11^{\text {th }}$ days the differences between all studied variants were leveled. Thus, it was established that the lectin activity in leaves and plant stems at drought conditions varies differently depending on the activity of inoculant strain.

Hence, performed studies have revealed that different water conditions affect lectin activity in different organs of alfalfa, which depend on the properties of inoculant strain, level of plant development and symbiosis formation. 
TABLE 2. Lectin activity $(\mathrm{OA} / 50 \mathrm{ml})$ in different organs of alfalfa under the different water supply conditions and inoculation with Sinorhizobium meliloti (experiment 2)

\begin{tabular}{|c|c|c|c|}
\hline \multirow[b]{2}{*}{ Variants } & \multicolumn{3}{|c|}{ Plant development stages } \\
\hline & $\begin{array}{c}\text { Shooting, } \\
3^{\text {rd }} \text { day of } \\
\text { draught }\end{array}$ & $\begin{array}{l}\text { Shooting, } \\
7^{\text {th }} \text { day of } \\
\text { draught }\end{array}$ & $\begin{array}{l}\text { Beginning of } \\
\text { budding, } \\
11^{\text {th }} \text { day of } \\
\text { draught }\end{array}$ \\
\hline & \multicolumn{3}{|c|}{ Leaves } \\
\hline $\begin{array}{l}\text { S.meliloti } \\
\text { CXM1-48, } 30 \% \\
\text { TMC }\end{array}$ & 8 & 2 & 2 \\
\hline $\begin{array}{l}\text { S.meliloti CXM1-48, } 60 \% \\
\text { TMC }\end{array}$ & 16 & 4 & 4 \\
\hline S.meliloti 441, 30\% TMC & 16 & 2 & 2 \\
\hline \multirow[t]{2}{*}{ S.meliloti $441,60 \% \mathrm{TMC}$} & 8 & 2 & 4 \\
\hline & \multicolumn{3}{|c|}{ Roots } \\
\hline $\begin{array}{l}\text { S.meliloti CXM1-48, } 30 \% \\
\text { TMC }\end{array}$ & 2 & 4 & 2 \\
\hline $\begin{array}{ll}\text { S.meliloti } & \text { CXM1-48, } \\
\text { TMC } & 60 \% \\
\end{array}$ & 2 & 2 & 0 \\
\hline S.meliloti 441, 30\% TMC & 2 & 4 & 2 \\
\hline \multirow{2}{*}{ S.meliloti $441,60 \% \mathrm{TMC}$} & 2 & 2 & 0 \\
\hline & \multicolumn{3}{|c|}{ Stems } \\
\hline $\begin{array}{l}\text { S.meliloti CXM1-48, } 30 \% \\
\text { TMC }\end{array}$ & 2 & 2 & 2 \\
\hline $\begin{array}{ll}\text { S.meliloti } & \text { CXM1-48, } \\
\text { TMC }\end{array}$ & 0 & 2 & 2 \\
\hline S.meliloti 441, 30\% TMC & 2 & 0 & 2 \\
\hline S.meliloti $441,60 \% \mathrm{TMC}$ & 0 & 0 & 2 \\
\hline
\end{tabular}

In the initial period of symbiosis formation (10-day old plant), the increase of lectin activity due to the insufficient water supply in alfalfa plants inoculated with active strain 441 was observed, whereas at plant inoculation with inactive strain SHM1-48 at remained unchanged. With start of biological nitrogen fixation (17-day old plants), the lectin activity in vegetative parts of alfalfa plants inoculated with inactive strain was higher than in variants with active rhizobia inoculation, remaining unchanged in plant roots. At this, no differences in variants with different water supply were noticed.

As the result of influence of water deficit on formed symbiosis (shooting beginning of budding stages), the changes of lectin activity in leaves and stems of alfalfa plants were determined by the activity of the inoculant strain, while no differences were observed in the roots under the use of both strains. 